39/2, julio-diciembre de 2009

pp. 539-554

ISSN 0066-5061

\title{
ASTRÓLOGOS Y ASTROLOGÍA AL SERVICIO DE LA MONARQUÍA NAVARRA DURANTE LA BAJA EDAD MEDIA (1350-1446) ${ }^{1}$
}

\author{
ASTROLOGERS AND ASTROLOGY \\ AT THE SERVICE OF NAVARRE'S MONARCHY \\ DURING THE LATE MIDDLE AGES (1350-1446)
}

\section{FERNANDO SERRANO LARRÁYOZ Universidad Pública de Navarra}

\begin{abstract}
Resumen: El artículo presenta una visión general de los astrólogos que sirvieron en la corte de Carlos II, Carlos III y Blanca I de Navarra. Un cargo cortesano que unía la adivinación de hechos futuros con la práctica médica del momento. Pese al prestigio que parecen alcanzar, no resulta una ocupación con cierta continuidad, salvo en casos muy concretos.
\end{abstract}

Palabras clave: Astrología-astronomía; Astrólogos; Medicina; Baja Edad Media; Navarra.

\begin{abstract}
This article provides a general view on astrologers who served at the courts of Carlos II, Carlos III and Blanca I of Navarre. This was a position that combined the foretelling of future events with the medical practice of the moment. Despite the prestige that astrologers seem to achieve, this position was never held long term except in some concrete cases.
\end{abstract}

Keywords: Astrology-astronomy; Astrologers; Medicine; Late Middle Ages; Navarre.

\section{SUMARIO}

1. Introducción.- 2. Astrólogos y astrología en la corte.- 3. Instrumentos y literatura astrológica en Navarra.- 4. Consideraciones finales.

\section{INTRODUCCIÓN}

Después de la medicina, una de las áreas de la ciencia medieval objeto de más elaboraciones y traducciones a lengua vulgar fue la relativa al

${ }^{1}$ Agradezco los comentarios al texto original realizados por Julio Samsó Moya, Lluís Cifuentes i Comamala, Juan Jesús Virto Ibáñez y Merche Osés Urricelqui. Igualmente, agradezco el tratamiento informático de las firmas de los astrólogos incluidas en el presente estudio a Fernando Bozano Garagorri. 
conocimiento de los astros y sus influencias. La astrología gozará, junto con la alquimia y la astronomía, de un inusitado interés, durante los últimos siglos medievales, entre los lectores de lenguas vernáculas de todo el Occidente latino, muy especialmente en el área mediterránea, incluido el reino de Navarra.

Para el hombre medieval, la astrología será vista como el instrumento más adecuado para proporcionar una visión lo más amplia y completa del ser humano, al permitir la comprensión de las interacciones entre el macrocosmos (disposición del universo) y el microcosmos (la vida en la Tierra). Un conocimiento que en la España cristiana se va perfilando en Cataluña a finales del siglo X, gracias al privilegiado contacto que tiene en ese momento con el mundo arabomusulmán. No obstante, el gran momento es el siglo XII, con centros en el valle del Ebro (Tudela, Tarazona) y Toledo. Por lo que respecta al siglo XIII destaca por el uso del castellano como lengua científica con el patrocinio de Alfonso X. Un periodo en que las traducciones del hebreo son escasas, frente a la importancia que alcanzan las del árabe. En los siglos XIV y XV, pese a la proliferación de tratados astrológicos y la mejora considerable del utillaje, no se dan grandes novedades, pero la difusión social de estos conocimientos llegó a ser masiva, en buena medida gracias al uso de las lenguas habladas.

Algunos autores han dejado bastante clara la íntima relación entre astronomía y astrología: el interés que suscita el estudio de la astrología - los conocimientos teóricos y los medios técnicos- fomenta el desarrollo de la astronomía durante y después del Renacimiento. Una astrología cuya utilización más básica fue la elaboración de horóscopos, que indicarían la influencia de los astros y planetas de una persona en el momento del nacimiento o en cualquier otro momento de la vida o en colectivos humanos, como por ejemplo las ciudades, lo que san Isidoro denominó astrologia supertitios $a^{2}$. Por estos horóscopos se podía saber dónde estaban ubicados los astros y los planetas en el momento del nacimiento de una persona, lo que

\footnotetext{
${ }^{2} \mathrm{La}$ ciencia medieval distingue dos tipos de astrología. Por un lado, la astrología iudiciaria, que trata de las influencias ocultas de los movimientos de los astros, del cambio que ocurre en las cúspides del horóscopo, o sea de las "casas angulares": ascendente, descendente, medio cielo y punto opuesto al medio cielo, y de la posición de los planetas en las "casas del horóscopo", en definitiva, sobre el destino del individuo y la sociedad. La astrología naturalis, por su parte, trata las influencias de esos movimientos sobre el cosmos y la naturaleza [Ron BARKAI, Significado de las aportaciones de los judíos en el terreno de la medicina, la astrología y la magia, en Ángel SÁENZ-BADILlos (ed.), Judios entre árabes y cristianos. Luces y sombras de una convivencia, Córdoba, El Almendro, 2000, p. 79].
} 
podía mostrar la influencia de los cuerpos celestes en su carácter y vida futura, pero también servía para conocer el momento concreto en el que practicar una actividad (viajes, matrimonios, intervenciones médicas o quirúrgicas...). En este último caso, lo importante no era la situación de los astros y los planetas en el momento del nacimiento del supuesto interesado, sino su posición en el momento presente o en un futuro inmediato.

Durante la Baja Edad Media la mayoría de las aplicaciones de la astrología no fueron motivo de controversia alguna, como en los casos de la influencia de los astros en el cuerpo humano y en la climatología, de manera que no existieron objeciones de importancia para su utilización en los procesos curativos o en la predicción del tiempo. Otra cosa fue su aceptación como forma de predecir el comportamiento humano, debido al peligro determinista que esto podía conllevar, contrario a la libertad individual. Si los astros podían gobernar los asuntos humanos, ¿no quedaba restringida la posibilidad del libre albedrío para el hombre y reducida la omnipotencia divina? Para salvar este obstáculo, los pensadores determinaron que la astrología podía predecir tendencias generales, pero no sucesos particulares; puesto que el libre albedrío del ser humano podía invalidar la influencia astral. Como reconoce Richard Kieckhefer, la base filosófica y científica de la astrología fue lo que le dio una posición respetable entre la intelectualidad europea. Así, pese a ser los astrólogos árabes quienes "refinaron" las bases filosóficas de la astrología, "utilizando argumentos eclécticos pero pseudo-aristotélicos para mostrar como la más perfecta quintaesencia de los cuerpos celestes podía ejercer poder sobre los cuerpos más pequeños de la Tierra", fue principalmente el apoyo de esta cosmología filosófica lo que permitió que la astrología ganara credibilidad en las universidades cristianas de la Europa occidental.

La utilización de la astrología afectó a la práctica de la medicina, considerando a la primera clave para permitir una nueva lectura de los problemas de la salud y la enfermedad. Como las incidencias celestes eran múltiples, se necesitaba conocer todas ellas y su influencia en el proceso patológico del paciente. Tanto es el interés que despierta esta práctica en el Occidente latino, pero muy especialmente en el área mediterránea, que su estudio se pasó a impartir en universidades como Salamanca, Bolonia o París, en donde en ocasiones se enseñaba la astronomía-astrología junto con la medicina. Un estímulo éste que afectó a la realeza, la nobleza y la alta burguesía, no sólo por motivos de salud sino también por lo apuntado anteriormente relacionado con las catástrofes de todo tipo, los cambios políticos, económicos, las guerras o hechos vitales de cualquier tipo. Una 
práctica, la de la astrología-astronomía, que igualmente conoció un importante desarrollo en las comunidades judías, en especial por la necesidad de profundizar en determinados fenómenos astronómicos que permitieran fijar con precisión el calendario litúrgico hebreo; motivos paralelos también se dieron en el caso de los árabes. Una ciencia en la que judíos de renombre, pero también cristianos y musulmanes, dejaron su impronta en las principales cortes reales peninsulares redactando y traduciendo obras de astronomíaastrología ${ }^{3}$.

\section{ASTRÓLOGOS Y ASTROLOGÍA EN LA CORTE}

Por lo que respecta a la presencia de astrólogos y la difusión de su ciencia en la corte navarra bajomedieval, puede parecer una temeridad intentar abordar este tema debido a la escasez de referencias documentales ${ }^{4}$. De hecho, no he hallado denominación alguna sobre astrólogos propiamente dichos hasta finales del siglo XIV. Una particularidad que me extraña teniendo en cuenta que en otras cortes limítrofes, como en la Corona de Aragón durante la primera mitad del siglo XIV, con Pedro IV (1336-1387), se comenzó una política de protección de sus astrólogos y de traducción al catalán de obras de astrología o redacción de obras originales sobre esta materia, continuada por

${ }^{3}$ Sobre lo dicho hasta el momento, véase Lluís CIFUENTES I COMAMALA, L'astronomia $i$ l'astrologia en català a finals de l'Edat Mitjana, en Antoni AMENGUA, Guillem Xabier PONS, Joan MARCH (eds.), Conferències de les Jornades de Commemoració i Estudi de l'eclipsi total de Sol a la Mallorca de 1905, Palma, Societat d' Història Natural de les Balears (Monografies 13), 2005, pp. 1-22; Lluís CIFUENTES I COMAMALA, La ciència en català a l'Edat Mitjana i el Renaixement, Barcelona-Palma de Mallorca, Universitat de Barcelona-Universitat de les Illes Balears, 2006 (segunda edición revisada y ampliada), pp. 189-192; José MANUEL GoNZÁLEZ SÁNCHEZ, Astrología y medicina: Pautas de investigación en las fuentes medievales, "Anuario de Estudios Medievales", 21 (1991), pp. 629-646; Enrique MonTERo Cartelle, De la Antigüedad a la Edad Media: Medicina, magia y astrología latinas, "Cuadernos del CEMYR", 8 (2000), pp. 53-72; Richard KIECKHEFER, La magia en la Edad Media, Barcelona, Crítica, 1992, pp. 130-142; Enrique CANTERA MONTENEGRO, Los judíos y las ciencias ocultas en la España medieval, "En la España Medieval”, 25 (2002), pp. 47-83; Roger FrENCH, Astrology in medical practice, en Luis GARCÍA BALLESTER, Roger FRENCH, Jon ARRIZABALAGA, y Andrew Cunningham (eds.), Practical Medicine from Salerno to Black Death, New York, Cambridge University, 1994, pp. 30-59; Nicolas WeILL-PAROT, Les "images astrologiques" au Moyen-Âge et à la Renaissance. Spéculations intellectuelles et pratiques magiques(XII $-X V^{e}$ siècle), París, Honoré Champion, 2002, pp. 439-588 (Para el caso concreto de la península Ibérica, véase pp. 772-780). Desde un punto de vista filosófico, véase el trabajo de Luis Miguel VICENTE GARCÍA, Una nueva filosofía de la astrología en los siglos XII y XIII: El impacto de las traducciones del árabe y a la postura de Santo Tomás de Aquino, "Revista Española de Filosofía Medieval", 9 (2002), pp. 249-264.

${ }^{4}$ Algunos datos sobre la presencia de astrólogos en la corte de Navarra, en Fernando SERRANO LARRÁYOZ, Medicina y enfermedad en la corte de Carlos III el Noble de Navarra (1387-1425), Pamplona, Gobierno de Navarra, 2004, pp. 104-106. 
Juan I (1387-1396) $)^{5}$. Las traducciones de obras de este tipo ya se venían haciendo en el reino de Castilla, sobre todo por el colectivo judío, como las famosas Tablas alfonsíes, editadas en 1212 bajo el auspicio de Alfonso X o también los Libros del saber de astronomía que tienen una finalidad claramente astrológica, así como otras obras alfonsíes: Libro Conplido, Libro de las Cruzes, la traducción del Tetrabiblos, etc. Recordemos también al nacido en Tudela (Navarra) Ibn Ezra, el más influyente de los judíos científicos altomedievales, autor de varios tratados astronómicos y astrológicos; a Yehudah ben Moshe ha-Kohén y Yishaq ibn Said (segunda mitad del siglo XIII), coautores de las mencionadas Tablas alfonsíes; y a Yehudah ben Aser y Yosef ibn Waqqãr, autores de sendas tablas astronómicas (segunda mitad del siglo XIV) ${ }^{6}$.

La falta de datos puede resultar engañosa a primera vista. Lluís Cifuentes i Comamala alude a la existencia de una serie de sencillos instrumentos utilizados por estos personajes - los astrólogos-, como los cuadrantes o relojes solares, útiles para determinar la hora a través de la posición del sol. La alusión en el registro del año 1350 a un tal "sol de comte de Foix" recibiendo de dono 50 sueldos del rey Carlos II (1349-1387), o a "maestre Pierres, tornantsol del Papa" recibiendo 12 libras, también de dono, en diciembre de 1387, por parte de Carlos III (1387-1425), puede que no se queden en meras coincidencias ${ }^{7}$. Debe tenerse en cuenta que la presencia de ambos "soles" en la corte coincide al poco de que los monarcas asciendan al trono. Ni que decir tiene que la alusión al término "Sol", el astro principal de

${ }^{5}$ Ll. Cifuentes i Comamala, La ciència en català, p. 191. Para la Corona de Aragón también pueden añadirse aquí los estudios de JoSEP M. Millàs VALLICROSA, Las Tablas Astronómicas del Rey Don Pedro el Ceremonioso [edición crítica de los textos hebraico, catalán y latino, con estudio y notas], Madrid-Barcelona, Consejo Superior de Investigaciones Científicas, 1962; y José CHABAS BERGón, Astronomía andalusí en Cataluña: las Tablas de Barcelona, en Josep CASUlleras y Julio SAMSÓ (eds.), From Baghdad to Barcelona: Studies in the Islamic exact sciencies in honour of Prof. Juan Vernet -De Bagdad a Barcelona: Estudios sobre historia de las ciencias exactas en el mundo islámico en honor del Prof. Juan Vernet, vol. 1, Barcelona, Instituto Millàs Vallicrosa de Historia de la Ciencia Árabe, 1996, pp. 477-525. En Portugal, por ejemplo, la presencia de astrólogos en la corte ya está documentada desde el siglo XII. Sobre todo se tiene constancia de su presencia y del interés por la astrología en las cortes de Alfonso IV (1325-1357), João I (1385-1433), Alfonso V (1449-1481), João II (1482-1495) o Manuel I (14951521) [Vasco Jorge RoSA DA SILvA, História da astronomia medieval portuguesa, Oporto, Ecopy, 2008, pp. 6-7].

${ }^{6}$ E. Cantera Montenegro, Los judíos y las ciencias, p. 69; José María Millás VAllicrosa, Estudios sobre historia de la ciencia española, Madrid, Consejo Superior de Investigaciones Científicas, 1991.

${ }^{7}$ Archivo General de Navarra (AGN), Comptos. Registros, $1^{\text {a }}$ Serie, $\mathrm{n}^{0}$ 61, fol. 111r; AGN, Comptos. Registros, $1^{\text {a }}$ Serie, $\mathrm{n}^{\mathrm{o}}$ 193, fol. $77 \mathrm{r}$. 
la órbita celeste, como un cargo cortesano, plantea la posibilidad de que sus labores en la corte tuvieran mucho que ver con las astrológicas, probablemente con una función más de tipo adivinatoria que médica, aunque pueden coincidir ${ }^{8}$. Sin embargo no son éstas las únicas referencias a este tipo de personajes. A principios de diciembre 1389 se recoge la presencia de otro de estos individuos, como "maestre Johan, lo sol del duc de Borgoyna", recibiendo un pequeño rocín'. En este caso la fecha tampoco parece casual, si se tiene en cuenta que el 13 de febrero de 1390 se iba a celebrar la coronación del monarca Carlos III. Con todo resulta evidente que todos ellos provienen de otras cortes señoriales: Foix, Aviñón y Borgoña, y su presencia en la de Navarra parece más o menos esporádica, incluso cuando empiezan a ser denominados como astrólogos propiamente, quizás, como se podrá comprobar más adelante, porque no suele ser infrecuente que en lo referente a cuestiones médicas algunos de los físicos que atendían a los monarcas también estuvieran versados en astrología.

Algunos años mas tarde, un alemán de origen y de reconocido prestigio en la corte de Juan I de Aragón ${ }^{10}$, maestre Reymar, "astrologuo" recibe, en 1396, 26 libras y 10 sueldos "de dono vna vez por pagar sus espensas que ha fechas en Esteilla despues que vino". Es probable que en este caso su estancia en la corte navarra sí que pueda atribuirse a labores sanadoras, ya que aparece recibiendo dicha cantidad en la misma nómina que el cirujano Sancho Sánchez de Isaba ${ }^{11}$. Durante ese año siguió cobrando toda una serie de donos y recompensas, como las 15 libras y 8 sueldos que se le entregaron para pagar dos sillas de montar, las 91 libras "en recompensation de vna otra mula que auia perdida en seruitio del rey" o las 152 libras, 7 sueldos y 6 dineros "por dono quito de $\mathrm{XX}^{\mathrm{no}}$ "12. Un año en el que bien pudieron servirle sus artes en la realización de horóscopos tras el nacimiento

\footnotetext{
${ }^{8}$ De conversaciones mantenidas con el profesor Julio Samsó, éste no tiene muy claro que el significado de "sol" o "tornantsol" tenga un significado astrológico. Por mi parte, hasta que no encuentre datos que lo desmientan, las circunstancias y las fechas en que aparecen documentados me permiten mantener esta hipótesis. Con todo, también debe tenerse en cuenta que en terminología alquímica "sol" equivale a "oro" y que tanto esos "soles" y muy particularmente el "tornantsol" también podrían ser alquimistas [Agradezco el dato a Lluís Cifuentes i Comamala].

${ }^{9}$ AGN, Comptos. Registros, $1^{\text {a }}$ Serie, $\mathrm{n}^{\mathrm{o}}$ 207, fol. 103r.

${ }^{10}$ Joseph María RocA, Johan I d'Aragó, Barcelona, Institució Patxot, 1929, pp. 224-225; Antoni CARDONER I PLANAS, Barcelona, Scientia, 1973, p. 271.

${ }^{11} \mathrm{AGN}$, Comptos. Documentos, caj. 72, n ${ }^{\circ}$ 6, 2 .

${ }^{12}$ AGN, Comptos. Documentos, caj. 75, $\mathrm{n}^{\mathrm{o}}$ 19, 9; AGN, Comptos. Registros, $1^{\text {a }}$ Serie, $\mathrm{n}^{\mathrm{o}}$ 233, fol. 43r y 46r; AGN, Comptos. Documentos, caj. 72, n ${ }^{\circ} 15,11$.
} 
de la infanta Isabel o en la boda de Juana, hermana bastarda del rey, al año siguiente. En 1397 forma parte de la comitiva que acompaña al rey a Francia, asignándosele un salario de 17 florines mensuales para mantener un valet y dos caballos, año en el que también nace el infante $\operatorname{Carlos}^{13}$.

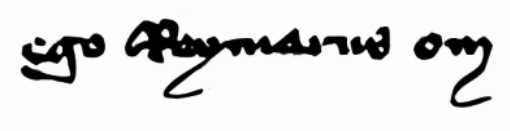

Firma autógrafa de Reymar, 1397

No es hasta 1410 cuando se justifica la presencia de otro astrólogo en la corte. Ahora se alude a un judío, Rabí Gento, que recibe 58 sueldos "por fazer sus expensas a yr d'Olit a Tudella do la reyna lo imbio por sus negocios" ${ }^{14}$. Nada más se sabe de él, ni los asuntos que le llevaron a la Ribera navarra. Seis años más tarde, concretamente el 3 de marzo, se tiene constancia de la presencia de un "maestre de astralabio", recibiendo del Hostal del rey un cuarto de carnero y 5 gallinas para su provisión ${ }^{15}$. De igual modo, mencionar que a finales de 1423 un tal Moisés Baco, judío y maestro en astrología, reconoce haber recibido 4 libras y 10 sueldos, por sus gastos en ofrecer sus servicios al monarca ${ }^{16}$. Sus conocimientos astrológicos se complementaban con los médicos, ya que al año siguiente aparece denominado como físico ${ }^{17}$. Parece que las ocupaciones para las que fue requerido este último debieron de ser puntuales, ya que posteriormente no vuelve a ser nombrado. Asimismo se desconoce con certeza su procedencia, aunque es probable que fuera originario de Tudela. Poco más puede decirse sobre él de lo ya comentado hasta ahora; tan sólo que es muy posible que a él fueran destinados "ciertos libros et otras cosas tocantes a estrologia" que el escudero real Martín de Ilúrdoz compró en 1423 por 30 florines de oro, una cantidad

\footnotetext{
${ }^{13} \mathrm{AGN}$, Comptos. Registros, $1^{\mathrm{a}}$ Serie, $\mathrm{n}^{\mathrm{o}} 240$, fol. 22v.

${ }^{14} \mathrm{AGN}$, Comptos. Registros, $1^{\text {a }}$ Serie, $\mathrm{n}^{\mathrm{o}} 309$, fol. $41 \mathrm{v}$.

${ }^{15} \mathrm{AGN}$, Comptos. Registros, $1^{\text {a }}$ Serie, $\mathrm{n}^{\mathrm{o}} 342$, fol. $487 \mathrm{v}$.

${ }^{16}$ AGN, Comptos. Documentos, caj. 108, $\mathrm{n}^{\circ} 15,82$.

${ }^{17} \mathrm{AGN}$, Comptos. Documentos, caj. 109, n ${ }^{\circ} 9,34$.
} 
nada despreciable ${ }^{18}$. Otro físico que muy bien pudo haber tenido conocimientos de astrología fue Juce Aboacar, físico del rey de Castilla, quien llegó a Navarra en la comitiva de la reina Leonor, en 1395, una vez solucionadas las desavenencias con su esposo ${ }^{19}$. Durante el tiempo que permanece en la corte se le menciona como físico de la reina, aunque también atiende al monarca cuando es necesario. Atendió a los reyes hasta finales de 1413, fecha en el que le sustituye su hijo Jacob, quien, en 1412, ya se encuentra al servicio del soberano recibiendo algunos donos. Quizás Juce (versión en romance de Yosef) pudiera tener algún tipo de vinculación, incluso familiar, con el ya aludido toledano Yosef ibn Waqqãr, quien por dos veces, entre 1357 y 1358 y entre 1395-1396, compiló una serie de tablas astronómicas ${ }^{20}$.

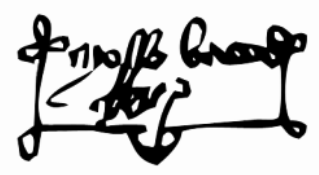

Firma autógrafa de Moisés Baco, 1423

Durante los primeros años del reinado de los reyes Blanca I y Juan II estuvo a su servicio un tal García Arnaldo de Suescun, aludido como "estorlogo del rey et de la reyna" o "estrologo et seruidor de la cambra de la seynora reyna". Una de las primeras referencias es de 1430, cuando recibe de los monarcas 6 libras "para en ayuda de quitar el estrelario" que tenía empeñado. Quizás por este motivo ese mismo año también recibe 60 sueldos de dono ${ }^{21}$. Dos años más tarde todavía la reina le debía ciertas cantidades por sus servicios:

Item a Garcia Arrnaut, astrollogo, semblanment por mandamiento de la dicha seynnora reynna por sus gages de ciertos tiempos que non le fueron

\footnotetext{
${ }^{18} \mathrm{AGN}$, Comptos. Documentos, caj. 108, $\mathrm{n}^{0}$ 16, 14.

${ }^{19}$ Sobre las cuestiones políticas que pudieron influir en la actitud de la corte castellana y de la propia reina, que había abandonado Navarra en 1388, véase Eloísa RAMíREZ VAQUERO, Carlos III, rey de Navarra. Príncipe de sangre Valois (1387-1425), Gijón, Trea, 2007, pp. 74-75.

${ }^{20} \mathrm{~F}$. Serrano Larráyoz, Medicina y enfermedad, pp. 55-57; David Romano, La ciencia hispanojudía, Madrid, Mapfre, 1992, pp. 161-162.

${ }^{21}$ AGN, Comptos. Registros, $1^{\text {a }}$ Serie, $\mathrm{n}^{0}$ 398, fol. 138; AGN, Comptos. Documentos, caj. $111, \mathrm{n}^{\mathrm{O}} 3,52$.
} 
contados en la escroa, et mandados pagar por gracia especial segunt mas largament paresce por el dicho mandamiento, la summa de trenta libras ${ }^{22}$.

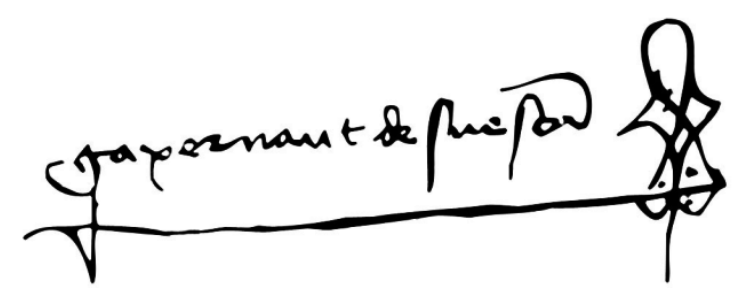

Firma autógrafa de García Arnaldo de Suescun, 1430

Poco más puede alcanzarse a conocer sobre la presencia de este tipo de "sabios" en la corte navarra. Se sabe que Carlos de Viana contó con alguno de ellos con posterioridad al fallecimiento de madre (1441). Así, el 12 de diciembre de 1446 un tal Juan Roser reconoce haber cobrado 9 libras por "sus trauaios a causa de cierta obra que yo fizi en adobar el extralauio por seruicio de su seynnoria" 23 .

\section{INSTRUMENTOS Y LITERATURA ASTRONOMICA-ASTROLÓGICA EN NAVARRA}

Si poco se alcanza a distinguir sobre los astrólogos al servicio real, mucho menos es lo que puedo aportar sobre los instrumentos y la literatura astronómica-astrológica en Navarra, salvo los ya comentados relojes solares y astrolabios como instrumentos de medida. Este último era una especie de "calculadora" portátil, que se utilizaba para medir la altura de los astros y resolver de esta manera los problemas de la astronomía esférica ${ }^{24}$. Se tiene

${ }^{22} \mathrm{AGN}$, Comptos. Documentos, caj. 134, 1, fol. $5 \mathrm{v}$.

${ }^{23}$ AGN, Comptos. Documentos, caj. 152, no 12, 59.

${ }^{24}$ Respecto a los instrumentos y a la literatura astrológica puede consultarse Emmanuel Poulle, Les instruments de la théorie des planètes selon Ptolomeée: équatoires et horlogerie planétaire du XIII ${ }^{e}$ au XVI ${ }^{e}$ siècle, 2 vols., Ginebra-París, Droz-Champion, 1982; y con un carácter más divulgativo y dirigido a la reconstrucción de modelos James E. MORRISON, The astrolabe, Rehoboth Beach, Janus, 2007. 
constancia del pago por la construcción en 1416, a un tal Juan de Santángelo, maestro en Artes, de un "estrument de astrología" para el servicio del monarca, probablemente correspondiese a una esfera armillar (o astrolabio esférico). Algo parecido al "estrelario" del referido García Arnaldo de Suescun, en el que participaron un buen número de plateros:

A Johan Garruayn, argentero que el rey li ha fecho dar et deliurar et ha puesto en dorar el estrument de astrologia que el rey ha fecho fazer pora su seruicio, 47 ducados d'oro (...). A Pascoalet d'Eguaras, tendero, morant en Ollit, por 2 libras, 11 oncas de argent viuo, et han seido compradas d'eill et distribuydos en el dorar del dicho instrument (...) 64 sueldos. A Nicholas d'Ezquioz por 9 libras de alum de cuba comprado d'eill et distribuydos en dorar el dicho instrument (...) 6 sueldos. A Rollet, argentero, por leynna, carbon et sal que ha comprado et expendido en dorar el dicho instrument (...) 22 sueldos. A maestre Johan de Sancto Archangelo, maestro en Artes, qui ha fecho el dicho instrument, por vna onça et media de plata que ha comprado et distribuydo en fazer figuras de estreillas et otros artifficios poral dicho instrument (...) 58 sueldos. Al dicho Johan Garbain por sus jornales de 32 dias que ha obrado en Ollit en dorar et drecar el dicho instrument, a 10 sueldos por dia, (...) vallen 16 libras. A Acach Açaya, judio, argentero de Pomplona, por sus jornales de otros 32 dias que ha obrado en Ollit a dorar del dicho instrument, a 8 sueldos por dia, (...) vallen 12 libras, 16 sueldos. A Martin d'Ochoui, a Rollet et a Johan de Thoro, argentero de Pomplona, que el rey lis ha fecho dar et deliurar semblament por sus jornalles de cada 19 dias que cada uno d'eillos han obrado en Ollit al dorar del dicho instrument, a 10 sueldos por dia a cada uno, (...) valen 38 libras, 10 sueldos $^{25}$.

Y si parcas son las fuentes relativas a los instrumentos utilizados por los astrólogos al servicio de los monarcas navarros, las alusiones a la literatura sobre astronomía-astrología no pasan de la mención a los libros adquiridos en 1423 por el escudero Juan de Ilúrdoz y la indicación de Juan Moliner, físico del Carlos III, fallecido en 1403, en cuyo testamento ordena la devolución de "dos libros que yo tengo de los predigadores d'Estella, de tablas vieias, que es el vno el Albert Maor (sic) et el otro Fisicas". Probablemente, y aunque tan solo sea una hipótesis, el primero podría corresponder al

\footnotetext{
${ }^{25}$ AGN, Comptos. Registros, $1^{\text {a }}$ Serie, $\mathrm{n}^{0} 344$, fol. 103. Se tiene constancia de que dicho Juan de Santángelo estuvo al servicio del monarca en 1415. En septiembre de ese año el monarca le asignó 14 sueldos y 6 dineros diarios por sus expensas, las de un mozo y dos rocines "por li fazer algunas obras a eill placenteras e que a su seruicio cumplian (...) ata tanto que eill aya acabado las dictas obras e el dicto seynnor rey li aya dado licencia" [José Ramón CASTRO, Carlos III el Noble, rey de Navarra, Pamplona, Institución Príncipe de Viana-Diputación Foral de Navarra, 1967, p. 524].
} 
Speculum astronomiae (1260) atribuido a Alberto Magno. Las “tablas viejas” evidentemente aluden a las cubiertas y pueden ser un indicio para retrotraer la datación de los volúmenes a una fecha (bastante) anterior a 1403. Un físico, Moliner, que también posee libros contrarios a la ortodoxia cristiana, como alguno de nigromancia (saberes con los que se pretende incidir en el futuro invocando a los demonios). Así, la posesión de libros de magia negra por parte de un médico ilustra muy bien la simbiosis de saberes en la época. De hecho deja muy claro el final que deben correr, probablemente influenciado por su confesor, fray Pedro de Eza:

Item mando et lexo al dicho Peyreton, mi fijo primogenito, todos mis libros de medicina que yo a present he en Navarra et quoalesquiere otros libros que yo he de ciencia, exceptado que si libros ningunos se trobaren entre aqueillos de nigromancia que aqueillos libros de nigromancia sean quemados por mano de mis cabeçaleros $(\ldots)^{26}$.

Sin embargo, la prueba más clara de que la literatura astronómicaastrológica no fue desconocida en Navarra es un bifolio conservado en el Archivo General de Navarra (sin catalogar), cuyo análisis paleográfico puede enmarcarse dentro de las góticas bastardas, utilizadas en los siglos XIV y $\mathrm{XV}^{27}$. En la Edad Moderna dicho pergamino debió de servir de cubierta de un libro perteneciente al gremio de zapateros y sastres de [Pamplona], si nos atenemos a la anotación, probablemente del siglo XVI: “de los calcateros y sastres" 28 . Fragmento de unas tablas en cuyos folios pueden leerse los títulos correspondientes: "Complimiento de la tabla del ygoalamiento de Venus" [fol. 1r], "Tabla del ygoalamiento de Mercurius" [fol. 1v], "Complimiento de la tabla del ygoalamiento de Saturnus" [fol. 2r], "Complimiento de la tabla del ygoalamiento de Saturnus" [fol. 2v]. Tablas de ecuaciones planetarias de

${ }^{26}$ Archivo Parroquial de San Cernin, Pergaminos, $\mathrm{n}^{0}$ 7. Sobre la astrología en la obra de Alberto Magno, véase Scott HENDRIX, Reading the Future and Freeing the Will: Astrology of the Arabic World and Albertus Magnus, "Hortulus: The Online Graduate Journal of Medieval Studies", 2/1(2006), pp. 30-49 [en línea] [http://hortulus.net/journal/20063Hendrix.pdf] [consulta: 5 de septiembre de 2008].

${ }^{27}$ Los rasgos de bastarda se aprecian sobre todo en los astiles de la letra "p", tienen un arranque grueso y conforme descienden el trazo se hace más fino, adquiriendo la punta de aguja, tan típica de las formas bastarda. Es una letra angulosa con contrastes entre los trazos gruesos y finos [Agradezco la información a la profesora Itziar Zabalza Aldave].

${ }^{28} \mathrm{Su}$ reutilización en un libro relacionado con el mundo de los oficios no parece permitir relacionar el códice en el que se incluyó las tablas con la casa real sino con estos ambientes, algo altamente interesante pues documentaría la difusión social de estos conocimientos fuera de los ambientes cortesanos y de las élites sociales o intelectuales. 
Saturno, Venus y Mercurio - tan sólo faltan Marte y Júpiter ${ }^{29}$ - que servían para calcular la longitud eclíptica del planeta, proceso que requería un complicado procedimiento de cálculos en el que intervienen los valores expuestos en estas tablas: "ygoalamiento del çentro" ("ecuación del centro") e "ygoalamiento del ypiciclo"("ecuación de la anomalía")

Una aproximación sobre dichas tablas realizada por el profesor Julio Samsó, a quien agradezco su colaboración, permite comprobar que la teoría sobre la que se basa es, sin duda, ptolemaica y las fuentes posibles son, o bien las Tablas de Toledo $^{31}$ o las Tablas de al-Battani ${ }^{32}$. Las columnas son las mismas que en las de al-Battani y de Toledo con la salvedad de que esta última fuente añade una columna final sobre la primera estación de cada planeta que deriva de las tablas de al-Jwarizmi-Maslama. Esta columna final no aparece en el bifolio, razón por la cual es más probable que la fuente sea al-Battani en el que esta columna tampoco aparece. Por el contrario, un factor que no confirma lo dicho hasta el momento es que la terminología de los cabezales no parece alfonsí. No estaría de más echar un vistazo a la traducción alfonsí de al-Battani conservada en un manuscrito de la Biblioteca del Arsenal en París. En último término tanto las de Toledo como las de al-Battani derivan de las Tablas Manuales de Teón de Alejandría, aunque hay que tener en cuenta el caso de Venus, ya que, como se considera que la excentricidad de Venus coincide con la del Sol, es frecuente que la ecuación del centro de

\footnotetext{
${ }^{29}$ Con la excepción del Sol y la Luna, durante la Baja Edad Media tan sólo se conocían cinco planetas o "estrellas movibles": Mercurio, Venus, Marte, Júpiter y Saturno. Véase V. J. RosA DA SILVA, História da astronomia, pp. 15-18.

${ }^{30}$ En el sistema ptolemaico, cada planeta se mueve gracias al juego coordinado de círculos, en el almagesto, y de anillos en las hipótesis planetarias. Esta complejidad de círculos es la que provoca la complejidad de los cálculos que deben aplicarse. Una de ellas es el deferente, un círculo cuyo centro se sitúa entre el ecuante y la Tierra. Otra esfera es el epiciclo, anclado en el deferente, y sobre el que se desplaza el planeta. El deferente es un círculo excéntrico con respecto a la Tierra, mientras el centro del epiciclo gira sobre el deferente, haciendo que el planeta se mueva más lejos o más cerca de la tierra en diferentes puntos de su órbita. Y haciendo también que pueda frenarse, detenerse e incluso retroceder (o retrogradar como se dice técnicamente). Estas son las correcciones que aparecen en las columnas "torçimiento lexos" y "torçimiento çerca". La hipotética disposición de las tablas (están sin foliación) viene dadas porque Mercurio y Venus son planeas inferiores y cuya posición se calcula de forma distinta a la de los planetas superiores, Saturno, Marte y Júpiter (faltan los dos últimos) [Agradezco estas precisiones a Julio Samsó y a Maria Mercè Viladrich Grau]. Un estudio completo sobre las características de algunas tablas astronómicas, en José CHABÁs y Bernard R. GolDSTEIN, The Alfonsine Tables of Toledo, Dordrecht-Boston-London, Kluwer Academic Publishers, 2003.

${ }^{31}$ Ed. crítica de Fritz Saaby PEDERSEN, The Toledan Tables. A review of the manuscripts and the textual versions with an edition, 4 vols., Copenhaguen, 2002.

${ }^{32}$ Ed. y trad. latina Carolo Alphonso NALLINO, Al-Battani sive Albatenii opus astronomicum. 3 vols. Milano, 1899-1907, Reprint Frankfurt, 1969; Hildesheim \& New York, 1977.
} 
Venus se adapte a la última determinación que se ha llevado a cabo de la excentricidad solar. Es difícil hacer este cotejo dado que conservamos menos de $45^{\circ}$ de la tabla de Venus. Por otra parte, en contra de la identificación de la posible fuente con las indicadas está el hecho de que tanto las Tablas de Toledo como las de al-Battani tabulan el argumento de grado en grado, mientras que en el bifolio navarro el argumento va de 30' en 30'. Con todo, no es imposible que, con intención de ser más preciso, el autor o el copista hubiera interpolado los valores para encontrar los intermedios.

El bifolio aludido contiene unas tablas de ecuaciones planetarias incompletas que se describen a continuación:

Fol. 1r (de acuerdo con la foliación que se le ha otorgado): Tablas de ecuaciones para Venus con argumentos desde 4 signos $15^{\circ} 30^{\prime}\left(=135^{\circ} 30^{\prime}\right)$ hasta 6 s. $0^{\circ}\left(=180^{\circ}\right)^{33}$.

Fol. 1v: Tablas de ecuaciones para Mercurio con argumentos desde 0 s. $0^{\circ} 30^{\prime}$ hasta 1 s. $15^{\circ}\left(=45^{\circ}\right)^{34}$.

Fol. 2r: Tablas de ecuaciones para Saturno con argumentos desde 1 s. $15^{\circ} 30^{\prime}=\left(45^{\circ} 30^{\prime}\right)$ hasta 3 s. $0^{\circ}\left(=90^{\circ}\right)^{35}$.

Fol. 2v: Siguen las tablas de ecuaciones para Saturno con argumentos desde $3 \mathrm{~s}$. $0^{\circ} 30^{\prime}$ hasta 4 s. $15^{036}$. Para finalizar, las columnas de las tablas corresponden (en terminología standard) a las siguientes funciones:

Columnas 1 y 2: "Argumentos" que corresponden (col. 1,a) a los que ha identificado antes y a los valores que respetan la simetría (col. 2, 360 a).

Columna 3: "Ecuación del centro", que permite corregir la longitud media del centro del epiciclo (calculada desde el centro del ecuante) para convertirla en longitud verdadera del centro del epiciclo (calculada desde el centro de la Tierra).

\footnotetext{
${ }^{33}$ Corresponde a Toledo, ed. F. S. PEDERSEN, IV, pp. 1295-1296, y a Battani, ed. C.A. NALLINO, II, pp. 130-131.

${ }^{34}$ Corresponde a Toledo, ed. F.S. PEDERSEN, IV, pp. 1300-1301, y a Battani, ed. C.A. NALLINO, II, pp. 132-133.

${ }^{35}$ Corresponde a Toledo, ed. F.S. PEDERSEN, IV, pp. 1268-1269, y a Battani, ed. C.A. NALLINO, II, pp. 109-110.

${ }^{36}$ Corresponde a Toledo, ed. F.S. PEDERSEN, IV, pp. 1270-1271, y a Battani, ed. C.A. NALLINO, II, pp. 111-112.
} 
Columna 4: "Minuta proporcionalia". Función de interpolación para ajustar la ecuación de la anomalía en función de la distancia del centro del epiciclo con respecto al apogeo planetario.

Columna 5: "Longitudo longior". Diferencia entre la ecuación de la anomalía en el apogeo y en la distancia media.

Columna 6: "Coequatio argumenti". Ecuación de la anomalía en la distancia media del centro del epiciclo

Columna 7: "Longitudo propior". Diferencia entre la ecuación de la anomalía en el perigeo y en la distancia media.

\section{CONSIDERACIONES FINALES}

Pese a los escasos datos existentes sobre el ejercicio de la astrologíaastronomía en la corte navarra bajomedieval, puede comprobarse que los monarcas no dudaron en utilizarla cuando lo consideraron necesario. Una presencia que de la documentación conservada se desprende como titubeante y muy puntual hasta finales del siglo XIV, periodo en el que están documentados no más de tres "soles" o adivinos adscritos a otras cortes, como Foix, Aviñón o Borgoña, con las que la corte navarra mantiene constantes relaciones. Es a partir de 1396 cuando se documenta la primera mención a un astrólogo propiamente dicho, de origen alemán y de reconocido prestigio en las cortes aragonesa y aviñonesa. Durante la primera mitad del siglo XV dos de los tres astrólogos expuestos son de origen judío, confirmando de este modo el reconocimiento que esta ciencia contaba en la comunidad hebrea. Sobre el último documentado, navarro y de origen cristiano, procede del otro lado de los Pirineos: de Suescun, en tierras de Ultrapuertos (o Baja Navarra). Por lo que respecta a la vertiente médica que tuvo la astrología-astronomía, se intuye que algunos físicos pertenecientes a la nómina de los hostales reales, sobre todo en el periodo de Carlos III el Noble, contaron con ciertos conocimientos astrológicos usuales en los que basar sus pronósticos y diagnósticos, siendo a la vez astrólogos.

Por lo que respecta al material científico utilizado, tanto el instrumental como la literatura existente, los ejemplos son muy escasos: astrolabios, relojes solares o unos indeterminados "instrumentos de astrología", como un "estrelario", probalemente una corrupción semántica de la palabra astrolabio. Algo semejante sucede con las alusiones a la literatura especializada, salvo vagas menciones a ciertos libros y la conservación de un fragmento de unas 
tablas astronómicas. Pese a la escasa documentación disponible, puede confirmarse que durante la Baja Edad Media Navarra, y muy especialmente su corte, estuvieron muy al corriente del interés que despertaban en el Occidente latino el conocimiento y la utilidad de la astrología. Así lo confirman los datos que se han podido recoger y, de forma muy significativa, el fragmento inédito de tablas astronómicas que se da a conocer en este trabajo.

Fecha de recepción del artículo: febrero 2009.

Fecha de aceptación y versión final: julio 2009. 


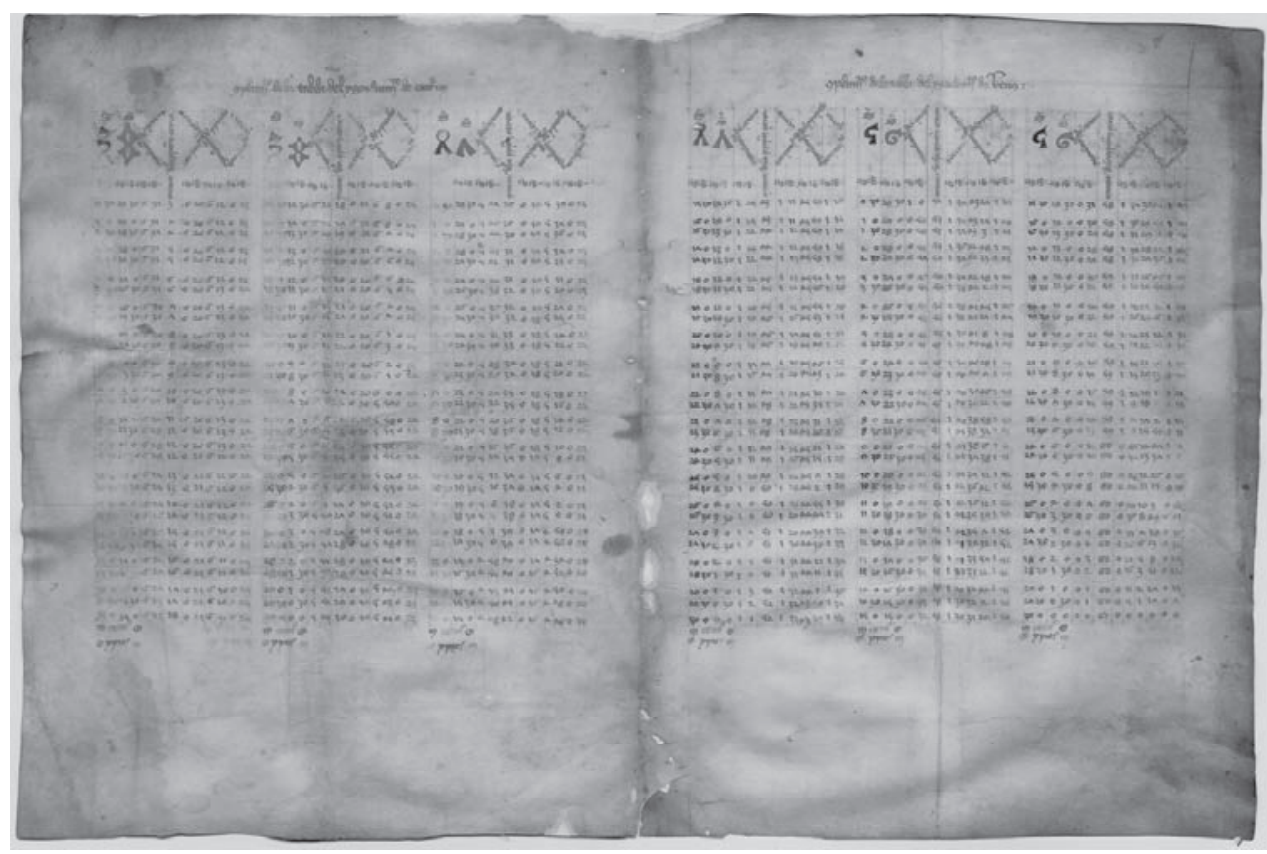

Tablas astronómicas (Siglos XIV-XV) [fol. 2v-1r]. Fotografía: Archivo General de Navarra

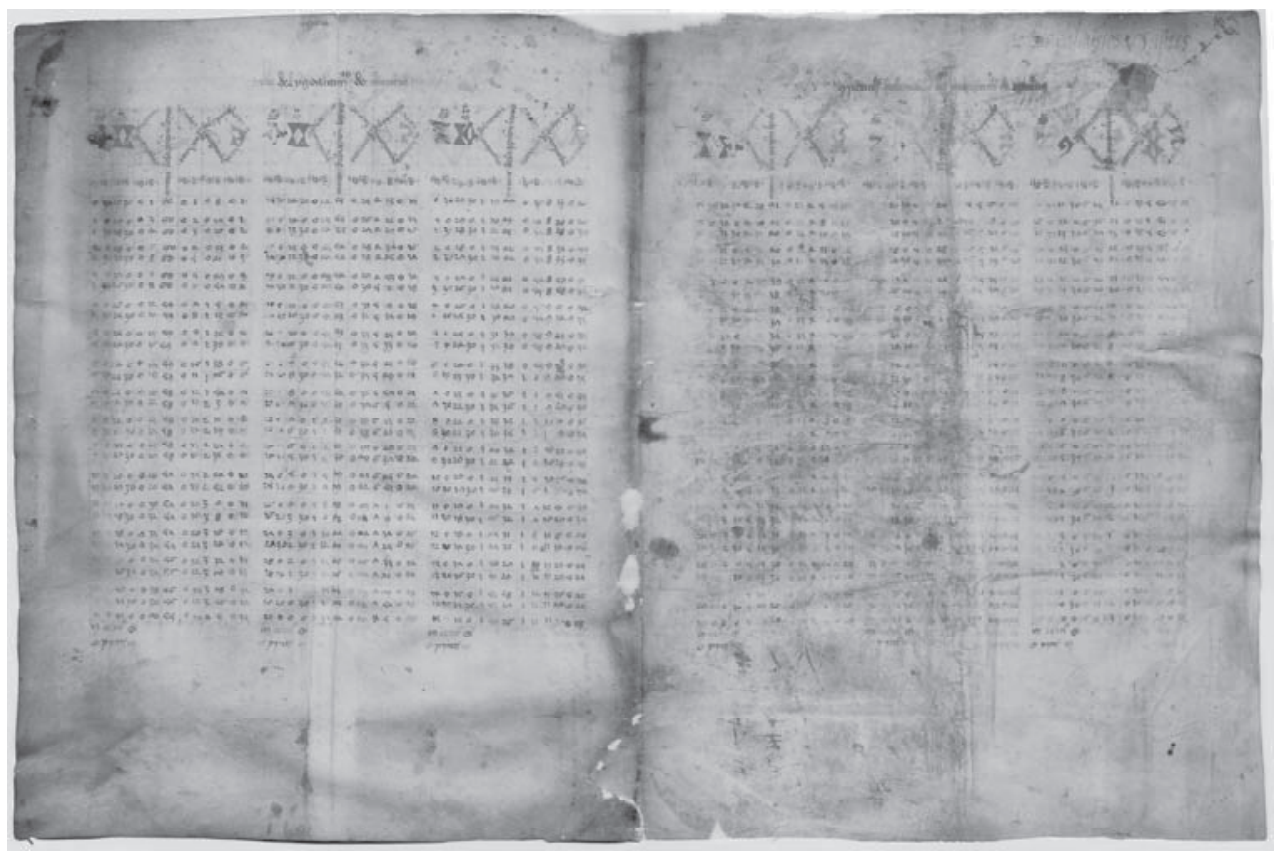

Tablas astronómicas (Siglos XIV-XV ) [fol. 1v-2r]. Fotografía: Archivo General de Navarra 